\title{
A CHARACTERIZATION OF 3-SPACE BY PARTITIONINGS
}

BY

\author{
R. H. BING
}

While it is apparent that any Euclidean $n$-sphere can be partitioned, it was shown only recently that any compact locally connected metric continuum is partitionable $\left({ }^{1}\right)$. In this paper it is shown how a 3 -sphere can be characterized in terms of its partitionings.

Definitions and notation. We designate Euclidean $n$-space by $E^{n}$. The unit $n$-sphere with center at the origin in $E^{n+1}$ is designated by $S^{n}$. A continuum is called a simple closed curve, simple surface (or 2-sphere), or 3-sphere according as it is topologically equivalent to $S^{1}, S^{2}$, or $S^{3}$. While a 3 -sphere may be regarded as the set of points in $E^{4}$ with coordinates $(x, y, z, w)$ satisfying $x^{2}+y^{2}+z^{2}+w^{2}=1$, we prefer to think of it as $E^{3}$ plus a point added in such a fashion that the exterior of a cube is topologically equivalent to its interior.

A simple surface $C$ in $E^{3}$ is called tame if there is a homeomorphism of $E^{3}$ into itself that carries $C$ into $S^{2}$. If there is no such homeomorphism, $C$ is called wild.

We shall suppose that space $S$ is metric, compact, locally connected, and connected.

A partitioning of $S$ is a collection of mutually exclusive open sets whose sum is dense in $S$. A sequence $G_{1}, G_{2}, \cdots$ of partitionings is a decreasing sequence of partitionings if $G_{i+1}$ is a refinement of $G_{i}$ and the maximum of the diameters of the elements of $G_{i}$ approaches 0 as $i$ increases without limit. A partitioning is regular if each of its elements is the interior of the closure of this element.

A regular partitioning $G$ is a brick partitioning if each element of $G$ is uniformly locally connected and the interior of the closure of the sum of each pair of elements of $G$ is uniformly locally connected. We know that $S$ has a decreasing sequence of brick partitionings $\left({ }^{2}\right)$.

The boundary of a set $A$ will be denoted by $F(A)$.

Characterization of a 3-sphere. A 3-sphere may be partitioned in many ways. The boundaries of some elements of a partitioning may be simple surfaces, the boundaries of others may be tori, and those of some may not even

Presented to the Society, December 27, 1949; received by the editors, January 31, 1950.

(1) R. H. Bing, Partitioning a set, Bull. Amer. Math. Soc. vol. 55 (1949) pp. 1101-1110, and E. E. Moise, Grille decomposition and convexification theorems for compact continua, Bull. Amer. Math. Soc. vol. 55 (1949) pp. 1111-1121.

(2) R. H. Bing, Complements of continuous curves, Fund. Math. vol. 36 (1950) pp. 303-318. 
be locally simply connected. However, a 3 -sphere has a partitioning in which all the elements of the partitioning have simple surfaces for boundaries. The following theorem gives a characterization of a 3 -sphere in terms of the manner in which it may be partitioned.

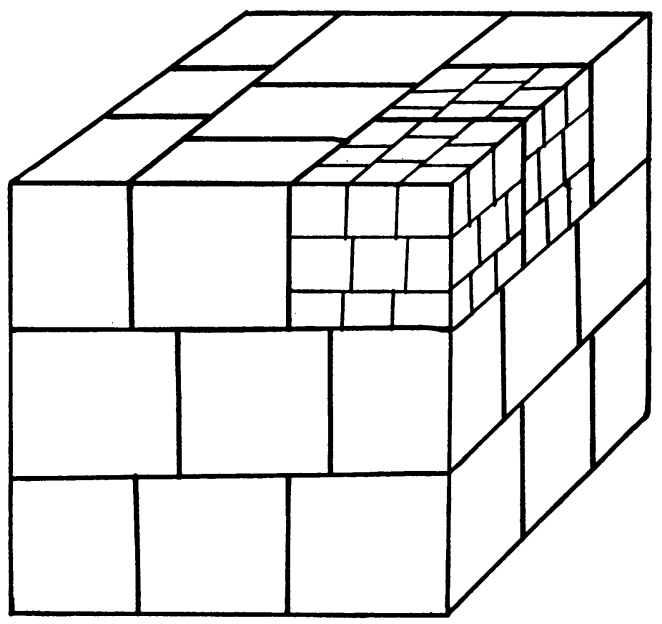

FIG. 1

THEOREM 1. A necessary and sufficient condition that $S$ be a 3-sphere is that one of its decreasing sequences of regular partitionings $G_{1}, G_{2}, \cdots$ have the following properties:

(1.1) The boundary of each element of $G_{i}$ is a simple surface.

(1.2) If the boundaries of two elements of $\sum G_{i}$ intersect, this intersection is a 2-cell.

(1.3) The intersection of the boundaries of three elements of $G_{i}$ is one-dimensional at each of its points.

(1.4) If $g$ is an element of $G_{i-1}(g=S$ if $i=1)$ the elements $G_{i}$ in $g$ may be ordered $g_{1}, g_{2}, \cdots, g_{n}$ so that $F\left(g_{j}\right)[j=1$ (if $\left.i>1), 2, \cdots, n\right]$ intersects $F(g)+F\left(g_{1}\right)+\cdots+F\left(g_{j-1}\right)$ in a connected set.

To see that the condition is necessary, one may consider the ways in which a cube may be filled with oriented rectangular solids all the same height. See Figure 1. Before showing that the condition is sufficient, we develop some other theorems to use. In Theorem 3 we show that there is a sequence of partitionings of $S^{3}$ that corresponds to $G_{1}, G_{2}, \ldots$ Using Theorem 5 we show further that there is such a sequence of partitionings of $S^{3}$ which is decreasing. The proof of the sufficiency in Theorem 1 follows Theorem 6.

The following is a combinatorial result. Although it will not be used in proving Theorem 1, it may be of interest for its own sake. 
THEOREM 2. If $C$ is a finite complex which is a 3-manifold without boundary, then $C$ is a 3-sphere if the 3-simplexes of $C$ may be ordered $c_{1}, c_{2}, \cdots, c_{n}$ such that $c_{j}(j=2, \cdots, n)$ intersects $c_{1}+c_{2}+\cdots+c_{j-1}$ in a connected set.

Proof. Consider $c_{i}$ to be a regular tetrahedron whose edges are of length 1 . Let $c_{i j}(j=1,2,3,4)$ be a regular tetrahedron in $c_{i}$ such that $c_{i j}$ has a vertex

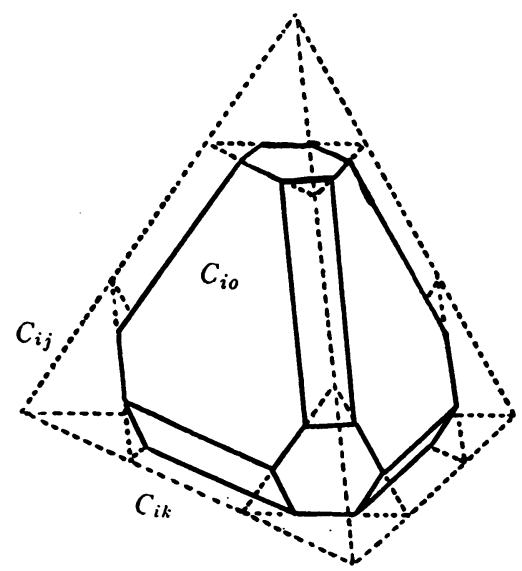

FIG. 2

in common with a vertex of $c_{i}$ and the edges of $c_{i j}$ are of length $1 / 3$. Let $c_{i k}(k=5,6, \cdots, 10)$ be a 5 -sided polyhedron in $c_{i}$ such that two faces of $c_{i k}$ are triangles which lie in faces of different $c_{i j}$ 's, two other faces are trapezoids of heights $1 / 9$ which lie in faces of $c_{i}$, and the fifth face is a rectangle whose interior lies in the interior of $c_{i}$. Let $c_{i 0}$ be the 14-sided polyhedron equal to the closure of $c_{i}-\left(c_{i 1}+c_{i 2}+\cdots+c_{i 10}\right)$. See Figure 2 .

Denote by $G$ the regular partitioning of $C$ where the closures of the elements of $G$ are of three types: (1) a $c_{i 0} ;(2)$ if $p$ is a vertex of a 3 -simplex in $C$, the sum of all $c_{i j}$ 's containing $p$; and (3) if $e$ is an edge of a 3 -simplex in $C$, the sum of all $c_{i k}$ 's having the middle third of $e$ as an edge.

Each element of $G$ is an open 3-cell whose boundary is a simple surface. To establish that elements of types 2 and 3 have these properties, one uses the hypothesis that $C$ is a 3 -manifold $\left({ }^{3}\right)$. If the boundaries of two elements of $G$ intersect, the intersection is a 2-cell. If the boundaries of three elements of $G$ intersect, the intersection is an arc. Furthermore, the elements of $G$ may be ordered $g_{1}, g_{2}, \cdots, g_{m}$ so that $F\left(g_{j}\right)$ intersects $F\left(g_{1}\right)+F\left(g_{2}\right)+\cdots$ $+F\left(g_{j-1}\right)$ in a connected set. To get such an ordering, suppose that $g^{\prime}$ precedes $g^{\prime \prime}$ if the first element of $c_{10}, c_{11}, \cdots, c_{10}, c_{20}, \cdots, c_{n 10}$ which is in $\bar{g}^{\prime}+\bar{g}^{\prime \prime}$ is in $\bar{g}^{\prime}$. Hence it follows from Theorem 3 that $C$ is a 3 -sphere.

THeоRem 3. Suppose $T$ is a homeomorphism carrying a simple surface $F_{0}$

(') H. Seifert and W. Threlfall, Lehrbuch der Topologie, New York, 1947, Theorem 1, p. 205. 
in $S$ into a tame simple surface $C$ in $E^{3}$, and $G$ is a regular partitioning of $S$ satisfying the following conditions:

(3.1) The boundary of each element of $G$ is a simple surface.

(3.2) If the boundaries of two elements of $G$ intersect, their intersection is the sum of a finite number of mutually separated 2-cells.

(3.3) The intersection of the boundaries of three elements of $G$ is one-dimensional at each of its points.

(3.4) $F_{0}$ is the boundary of the closure of the sum of an ordered subcollection $\left[g_{1}, g_{2}, \cdots, g_{n}\right]$ of $G$ such that $F\left(g_{j}\right)(j=1,2, \cdots, n)$ intersects $F_{0}+F\left(g_{1}\right)$ $+\cdots+F\left(g_{j-1}\right)$ in a connected set.

Then there is a partitioning $\left[h_{0}, h_{1}, \cdots, h_{n}\right]$ of $E^{3}$ and a homeomorphism $T^{\prime}$ of the boundary (in $S$ ) of $g_{1}+g_{2}+\cdots+g_{n}$ into the boundary (in $E^{3}$ ) of $h_{1}$ $+h_{2}+\cdots+h_{n}$ such that $h_{0}$ is the exterior of $C, F\left(h_{i}\right)$ is a tame simple surface, $T=T^{\prime}$ on $F_{0}$, and $T^{\prime}\left[F\left(g_{i}\right)\right]=F\left(h_{i}\right)$.

Proof. We shall use induction on $n$. If $n=1$, the theorem is true because we can let $h_{1}$ be the interior of $C$ and $T^{\prime}$ be $T$.

If $p$ is an interior point of a 2 -cell $K$ (interior with respect to $K$ ) which lies in the intersection of the boundaries of two elements $g_{i}, g_{j}$ of $G, p$ is not on the boundary of any other element $g_{r}$ of $G$ or else $F\left(g_{i}\right) \cdot F\left(g_{r}\right)$ would contain a 2 -cell intersecting $p$. But this is impossible since both $K$ and $F\left(g_{i}\right) \cdot F\left(g_{r}\right)$ lie on the simple surface $F\left(g_{i}\right)$, and $F\left(g_{r}\right) \cdot K$ is prevented by condition (3.3) from being two-dimensional. Hence, the common part of the boundaries of three elements of $G$ is a subset of the boundaries of the sum of the 2-cells which is the common part of some two of them. Hence, condition (3.3) may be replaced by the following condition.

$(3.3)^{\prime}$ if the boundaries of three elements of $G$ intersect, their intersection is the sum of a finite number of arcs.

We shall show that $\bar{g}_{1}+\bar{g}_{2}+\cdots+\bar{g}_{n}-F_{0}$ is connected. If it were the sum of two mutually separated sets $H$ and $K, F_{0}$ would be the boundary of $H+K$ and each of the sets $\bar{H}, \bar{K}$ would intersect $F_{0}$. Then $\bar{H} \cdot \bar{K}$ would contain a point $p$ of $F_{0}$. There would be elements $g_{i}$ and $g_{j}$ of $\left[g_{1}, g_{2}, \cdots, g_{n}\right]$ in $H$ and $K$ respectively such that $\bar{g}_{i} \cdot \bar{g}_{j}$ contains $p$. It follows from (3.3) that $F_{0} \cdot F\left(g_{i}\right)$ - $F\left(g_{j}\right)$ is one-dimensional and from (3.2) that $F\left(g_{i}\right) \cdot F\left(g_{j}\right)$ contains a 2-cell. Hence $H$ and $K$ could not be mutually separated because the points of $\bar{g}_{i} \cdot \bar{g}_{j}$ not in $F_{0}$ would be limit points of $H$ and $K$.

We now show that if $n \geqq 2$, then $F_{0} \cdot F\left(g_{1}\right)$ is topologically equivalent to a bounded subset of the plane whose boundary is the sum of a finite number of mutually exclusive circles. Since $F\left(g_{1}\right)$ is a simple surface which contains a point not of $F_{0}, F_{0} \cdot F\left(g_{1}\right)$ is a proper subset of $F_{0}$. From condition (3.4) we find that $F_{0} \cdot F\left(g_{1}\right)$ is connected. Furthermore, it follows from condition (3.2) that $F_{0} \cdot F\left(g_{1}\right)$ is the sum of a finite number of 2-cells each of which is a component of the intersection of $F\left(g_{1}\right)$ and the boundary of an element of $G$ not 
in $\left[g_{1}, g_{2}, \cdots, g_{n}\right]$. As a result of condition $\left(3.3^{\prime}\right)$, if two of these 2-cells intersect, their common part is the sum of a finite number of arcs. Hence, $F_{0} \cdot F\left(g_{1}\right)$ is topologically equivalent to a bounded subset of the plane whose boundary is the sum of a finite collection of mutually exclusive circles.

Let $J_{1}, J_{2}, \cdots, J_{t}$ be the components of the boundary with respect to $F_{0}$ of $F_{0} \cdot F\left(g_{1}\right), I_{i}(i=1,2, \cdots, t)$ be the component of $F_{0}-J_{i}$ containing no point of $F\left(g_{1}\right)$, and $K_{i}$ be the component of $F\left(g_{1}\right)-J_{i}$ containing no point of $F_{0}$. The boundary of $\bar{g}_{2}+\bar{g}_{3}+\cdots+\bar{g}_{n}$ is $\sum\left(I_{i}+J_{i}+K_{i}\right)$ as can be shown from conditions (3.1), (3.2), and (3.3). However, no component of $\bar{g}_{2}$ $+\bar{g}_{3}+\cdots+\bar{g}_{n}$ intersects two elements of $\left\{I_{i}+J_{i}+K_{i}\right\}$. If this were not true there would be an integer $j$ such that $\bar{g}_{2}+\bar{g}_{3}+\cdots+\bar{g}_{j}$ contains a continuum intersecting two elements of $\left\{I_{i}+J_{i}+K_{i}\right\}$ but $\bar{g}_{2}+\bar{g}_{3}+\ldots$ $+\bar{g}_{j-1}$ contains no such continuum. However, $F\left(g_{j}\right)$ would not intersect $F_{0}+F\left(g_{1}\right)+\cdots+F\left(g_{j-1}\right)$ in a connected set as required by condition (3.4). Hence, each component of $\bar{g}_{2}+\bar{g}_{3}+\cdots+\bar{g}_{n}$ has one of the simple surfaces of $\left\{I_{i}+J_{i}+K_{i}\right\}$ for a boundary.

If $H_{i}(i=1,2)$ is a collection of $t$ mutually exclusive simple closed curves on a simple surface such that no element of $H_{i}$ separates any other two elements of $H_{i}$ from each other on the surface, there is a homeomorphism of the surface into itself that carries the elements of $H_{1}$ into the elements of $H_{2}$. Also, for each homeomorphism $T^{\prime \prime}$ of the surface of a cube in $E^{3}$ into itself, there is a homeomorphism of $E^{3}$ into itself that preserves $T^{\prime \prime}$ on the surface of the cube. Hence, if $T$ is the transformation mentioned in the statement of Theorem 3, without loss of generality we may suppose that $C$ is the surface of a cube and the $T\left(J_{1}\right), T\left(J_{2}\right), \cdots, T\left(J_{t}\right)$ are circles $C_{1}, C_{2}, \cdots, C_{t}$ all lying in one base of $C$. Let $W_{i}(i=1,2, \cdots, t)$ be the hemispherical surface which contains $C_{i}$ and lies in $C$ plus its interior. There is a homeomorphism $T_{i}$ of $K_{i}$ into $W_{i}$ such that if $p$ is a point of $J_{i}, T(p)=T_{i}(p)$. Suppose $T^{\prime}(p)$ $=T(p)$ if $p$ is a point of $F_{0}$ and $T^{\prime}(p)=T_{i}(p)$ if $p$ is a point of $K_{i}$. Then $T^{\prime}$ is a homeomorphism carrying $F_{0}+F\left(g_{1}\right)$ into $C+\sum W_{i}$.

Each bounded complementary domain of $C+\sum W_{i}$ is a 3-cell and the boundary of each of these domains is tame and the image under $T^{\prime}$ of either $F\left(g_{1}\right)$ or the boundary of a component of $\bar{g}_{2}+\bar{g}_{3}+\cdots+\bar{g}_{n}$. By induction on $n$, the complementary domains of $C+\sum W_{i}$ can be partitioned and the homeomorphism $T^{\prime}$ extended on the boundaries of these partitionings so that a partitioning $\left[h_{0}, h_{1}, \cdots, h_{n}\right]$ and a homeomorphism $T^{\prime}$ are obtained which satisfy the conditions of the theorem.

Theorem 4. Suppose $C$ is a tame simple surface in $E^{3}$ and $G_{1}, G_{2}, \cdots$ is a sequence of partitionings of $E^{3}$ satisfying the following conditions:

(4.1) The boundary of each element of $G_{i}$ is a tame simple surface.

(4.2) If $g$ is an element of $G_{i}$ whose closure intersects $C, F(g) \cdot C$ is connected and does not separate $F(g)$.

(4.3) Each $G_{i+1}$ is a refinement of $G_{i}$. 
(4.4) One element of $G_{i}$ is the exterior of $C$.

(4.5) For each positive number $\epsilon$ there is a positive integer $n(\epsilon)$ such that the closure of no element of $G_{n}$ other than the exterior of $C$ contains two points which belong to the boundary of the sum of the elements of $G_{1}$ and are farther apart than $\epsilon$.

Let $K$ be a closed set and $R$ be a closed proper subset of $C$ such that if $g$ is an element of $G_{1}$ other than the exterior of $C$, then $F(g) \cdot C$ does not intersect both $R$ and $K$. Then there are an integer $m$ and a homomeomorphism $T$ of $E^{3}$ into itself satisfying the following conditions:

(4.6) Each point of the boundary of an element of $G_{1}$ is invariant under $T$.

(4.7) If $g$ is an element of $G_{m}$ other than the exterior of $C, T(\bar{g})$ does not intersect both $R$ and $K$.

Proof. Let $g_{1}, g_{2}, \cdots, g_{j}$ be the elements of $G_{1}$ that lie on the interior of $C$ and whose boundaries intersect $R$. Let $J_{i}$ be a simple closed curve on $F\left(g_{i}\right)$ such that one complementary domain $I_{i}$ of $J_{i}$ in $F\left(g_{i}\right)$ contains $F\left(g_{i}\right) \cdot R$ but $I_{i}+J_{i}$ does not contain a point of $K$. It follows from condition (4.5) that there is an integer $m$ such that if $g$ is an element of $G_{m}$ other than the exterior of $C$, the intersection of $\bar{g}$ with the boundary of the sum of the elements of $G_{1}$ is a subset of $I_{1}+I_{2}+\cdots+I_{j}$ if it intersects $R$.

There is a homeomorphism $T_{i}$ carrying $\bar{g}_{i}$ into itself that leaves each point of $F\left(g_{i}\right)$ invariant and such that if $g$ is an element of $G_{m}$ in $g_{i}$ and $\bar{g}$ intersects $R$, then $T_{i}(\bar{g})$ does not intersect $K$. (To see that this is so, think of $\bar{g}_{i}$ as a cube plus its interior whose base is $I_{i}+J_{i}$. Then $T_{i}$ is a homeomorphism that moves each point of $g_{i}$ toward the base.) Then the required transformation $T$ satisfying conditions (4.6) and (4.7) is one that is the identity except on $g_{1}+g_{2}+\cdots+g_{j}$ and is $T_{i}$ on $g_{i}$.

Theorem 4 could be improved by replacing $G_{i}$ by $G_{1}$ in conditions (4.1), (4.2), and (4.3) but the stronger conditions as stated are needed in Theorem 5.

Theorem 5. Suppose that $C$ is a tame simple surface in $E^{3}$ and $G_{1}, G_{2}, \ldots$ is a sequence of partitionings of $E^{3}$ satisfying conditions (4.1), (4.2), (4.3), and (4.4) as well as the following:

(5.1) For each positive integer $j$ and each positive number $\epsilon$ there is a positive integer $n(j, \epsilon)$ such that the closure of no element of $G_{n}$ other than the exterior of $C$ contains two points of the boundary of the sum of the elements of $G_{j}$ which are farther apart than $\epsilon$.

Then for each positive number $\delta$, there is an integer $m$ and a homeomorphism $T$ of $E^{3}$ into itself such that $T$ leaves each point of the exterior of $C$ invariant and $T$ carries each other element of $G_{m}$ into a set of diameter less than $\delta$.

We could not substitute for condition (4.2) one like condition (3.2) of Theorem 3.

If we tried to make such a substitution, we could not take care of the case where two mutually exclusive 2-cells $D_{1}$ and $D_{2}$ on $C$ are "hooked together" 
by two elements of $G_{1}$, by four elements of $G_{2}$, by eight elements of $G_{3}, \cdots$ as illustrated in Figure 3. For the sake of clarity, only the elements of $G_{1}$ which "hook" $D_{1}$ and $D_{2}$ together are shown in the diagram but if $g_{1}$ and $g_{2}$ are these two elements, one may see how the other elements of $G_{1}$ can be placed by considering a homeomorphism carrying $C$ plus its interior, $\bar{g}_{1}$, and $\bar{g}_{2}$ into the oriented rectangular solids with opposite vertices at $(0,0,0)$ and $(1,1,1)$, $(2 / 5,0,1 / 5)$ and $(3 / 5,1,2 / 5)$, and $(2 / 5,0,3 / 5)$ and $(3 / 5,1,4 / 5)$ respectively.

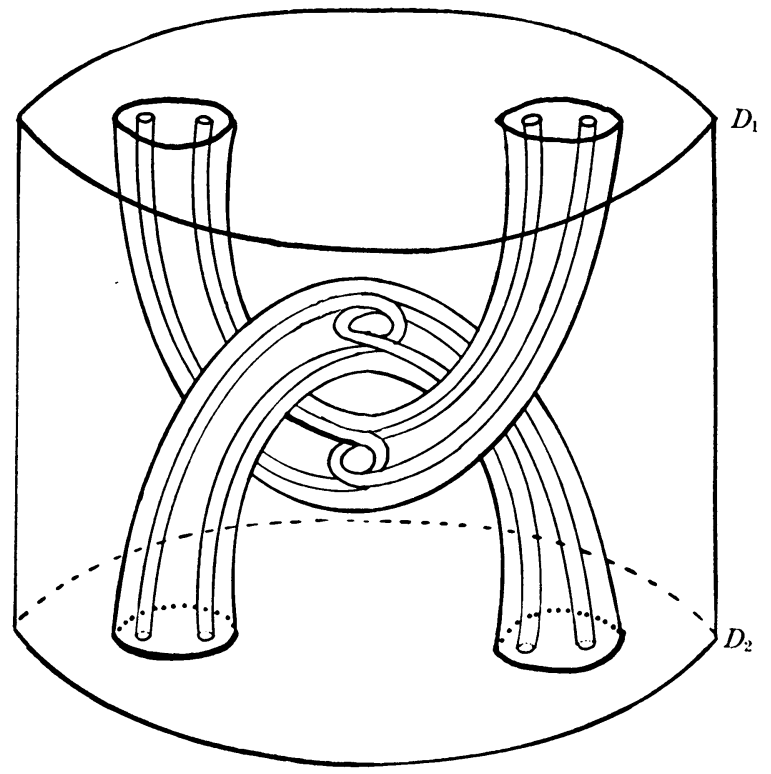

FIG. 3

Proof of Theorem 5. For convenience we suppose that $C$ is the surface of a cube oriented with the $x, y$, and $z$ axes. (If it is not one, there is a homeomorphism $T_{0}$ of $E^{3}$ into itself that carries it into one. If the elements of $G_{i}$ are replaced by their images, conditions (4.1), (4.2), (4.3), (4.4), and (5.1) still hold. Also, there is a positive number $\delta^{\prime}$ such that if $p$ and $q$ are two points of $T_{0}\left(C+\right.$ its interior) whose distance apart is less than $\delta^{\prime}$, then $T_{0}^{-1}(p)$ and $T_{0}^{-1}(q)$ are no farther apart than $\delta$.)

Let $n$ be an integer so large that if $g$ is an element of $G_{n}$ other than the exterior of $C, F(g)$ does not intersect $C$ in two points that are farther apart than $\delta / 12$. Let $S_{1}$ be a single-valued surface defined above the base of $C$ such that each point of $S_{1} \cdot C$ is $\delta / 4$ units above the base of $C$.

We find from Theorem 4 that there is an integer $n_{2}$ and a homeomorphism $T_{2}$ of $E^{3}$ into itself such that (1) $T_{2}$ leaves invariant each point of the boundary of the sum of the elements of $G_{n}$ and (2) if $g$ is a bounded element of $G_{n_{2}}$, $T_{2}(\bar{g})$ does not intersect both $S_{1}$ and a point of $C$ whose distance is as much 
as $2 \delta / 4$ from the base of $C$. In this application of Theorem 4 we let $K$ and $R$ in the statement of Theorem 4 be $S_{1}$ and the points of $C$ which are no nearer the base of $C$ than $2 \delta / 4$. There is a single-valued surface $S_{2}$ above the base of $C$ and $S_{1}$ such that (1) each point of $S_{2} \cdot C$ is $2 \delta / 4$ units above the base of $C$ and (2) $S_{2}$ does not intersect the image under $T_{2}$ of an element of $G_{n_{2}}$ that intersects $S_{1}$. To ensure that (2) will be satisfied, we need only take $S_{2}$ sufficiently close to the portion of $C$ that lies above $S_{2}$.

In a second application of Theorem 4 we let $C, G_{1}, K$, and $R$ in the statement of Theorem 4 be $C$, the collection of images under $T_{2}$ of the elements of $G_{n_{2}}, S_{2}$, and the set of points of $C$ which are not nearer the base of $C$ than $3 \delta / 4$. We find that there is an integer $n_{3}$ and a homeomorphism $T_{3}$ of $E^{3}$ into itself such that (1) $T_{3}$ preserves $T_{2}$ on the sum of the boundaries of the elements of $G_{n_{2}}$, and (2) if $g$ is a bounded element of $G_{n_{3}}, T_{3}(\bar{g})$ does not intersect both $S_{2}$ and a point of $C$ whose distance is as much as $3 \delta / 4$ from the base of $C$. There is a single-valued surface $S_{3}$ between $S_{2}$ and the top of $C$ such that (1) each point of $S_{3} \cdot C$ is $3 \delta / 4$ units above the base of $C$ and (2) the image under $T_{3}$ of no element of $G_{n_{3}}$ intersects both $S_{2}$ and $S_{3}$.

Similarly there are mutually exclusive surfaces $S_{3}, S_{4}, \cdots, S_{j}(j \delta / 4$ < height of $C \leqq(j+1) \delta / 4)$, an integer $n_{j}=n_{z}$, and a homeomorphism $T_{j}$ of $E^{3}$ into itself such that (1) $S_{i}$ is single-valued above the base of $C$, (2) each point of $S_{i} \cdot C$ is $i \delta / 4$ units above the base of $C$, (3) $T_{j}$ leaves invariant each boundary point of each element of $G_{n}$, and (4) no image under $T_{j}$ of an element of $G_{n_{2}}$ intersects two of the surfaces $S_{1}, S_{2}, \cdots, S_{j}$.

There is a homeomorphism $T^{\prime}$ of $E^{3}$ into itself which leaves each point of $C$ invariant, which carries $S_{1}, S_{2}, \cdots, S_{j}$ into surfaces parallel to the base of $C$ and such that the image of a point under $T^{\prime}$ has the same $x$ and $y$ values as the point has. Then the transformation $T^{\prime} T_{j}=T_{z}$ is one which (1) leaves invariant each point of $C$, (2) does not move any point of the boundary of the sum of the elements of $G_{n}$ in the $x$ or $y$ directions, and (3) if $p$ and $q$ are two points of a bounded element of $G_{n_{z}}$, the $z$ coordinates of $T_{z}(p)$ and $T_{z}(q)$ do not differ by more than $\delta / 2$.

Similarly, we find that there is a homeomorphism $T_{y}$ of $E^{3}$ into itself and an integer $n_{y}$ greater than $n_{z}$ such that (1) $T_{y} T_{z}$ leaves invariant each point of $C$ plus its exterior, (2) if $p$ is a point of the boundary of an element of $G_{n_{s}}$, $T_{y} T_{z}(p)$ has the same $x$ and $z$ coordinates as $T_{z}(p),(3)$ if $p$ and $q$ are two points of the same bounded element of $G_{n_{y}}$ the $y$ coordinates of $T_{y} T_{z}(p)$ and $T_{y} T_{z}(q)$ do not differ by more than $\delta / 2$. Also, there is an integer $n_{x}$ greater than $n_{y}$ and a homeomorphism $T_{x}$ of $E^{3}$ into itself such that (1) $T_{x} T_{y} T_{z}$ leaves invariant each point of $C$ plus its interior, (2) if $p$ is a point of the boundary of an element of $G_{n_{y}}, T_{x} T_{y} T_{z}(p)$ has the same $y$ and $z$ coordinates as $T_{y} T_{z}(p)$, and (3) if $p$ and $q$ are two points of the same bounded element of $G_{n_{x}}$, then the $x$ coordinates of $T_{x} T_{y} T_{z}(p)$ and $T_{x} T_{y} T_{z}(q)$ do not differ by more than $\delta / 2$. 
If $p$ and $q$ are two points of a bounded element of $G_{n_{x}}$, the $y$ coordinates of $T_{x} T_{y} T_{z}(p)$ and $T_{x} T_{y} T_{z}(q)$ do not differ by more than $\delta / 2$ because $p$ and $q$ belong to an element of $G_{n_{y}}$, the $y$ coordinates of $T_{y} T_{z}(p)$ and $T_{y} T_{z}(q)$ do not differ by as much as $\delta / 2$, and $T_{x}$ does not alter the length in the $y$ direction of an element of $G_{n_{y}}$. Furthermore, we find that if $p$ and $q$ are two points of a bounded element of $G_{n_{x}}$, the $z$ coordinates of $T_{x} T_{y} T_{z}(p)$ and $T_{x} T_{y} T_{z}(q)$ do not differ by more than $\delta / 2$. Hence, the image under $T_{x} T_{y} T_{z}$ of each bounded element of $G_{n_{x}}$ has a diameter of less than $\delta$. Then $n_{x}$ is the integer and $T_{x} T_{y} T_{z}$ the transformation required in Theorem 5 .

THEOREM 6. The compact continua $M_{1}$ and $M_{2}$ are homeomorphic if there is a decreasing sequence of partitionings $G_{i 1}, G_{i 2}, \cdots(i=1,2)$ for $M_{i}$ and $a$ 1-1 correspondence between the elements of $G_{1 j}$ and $G_{2 j}$ such that (1) two elements of $G_{1 j}$ have a boundary point in common if and only if the corresponding elements of $G_{2 j}$ have a boundary point in common and (2) corresponding elements of $G_{1 j+1}$ and $G_{2 j+1}$ are subsets of corresponding elements of $G_{1 j}$ and $G_{2 j}$.

Proof of sufficiency in Theorem 1. Suppose that the ordering of $G_{1}$ mentioned in condition (1.4) is $g_{1}, g_{2}, \cdots, g_{n}$. We first show that $\bar{g}_{1}$ is topologically equivalent to a 3 -cell.

Let $C$ be the surface of a cube in $E^{3}$ and $T_{1}$ be a homeomorphism carrying $F\left(g_{1}\right)$ into $C$. We find from Theorem 3 that there is a sequence of partitionings $H_{1}, H_{2}, \cdots$ of $E^{3}$ and a sequence of homeomorphisms $T_{1}, T_{2}, \cdots$ satisfying the following:

(1.5) The boundary of each element of $H_{i}$ is a tame simple surface.

(1.6) $H_{i+1}$ is a refinement of $H_{i}$.

(1.7) One element of $H_{i}$ is the exterior of $C$.

(1.8) There is a 1-1 correspondence between the bounded elements of $H_{i}$ and the elements of $G_{i}$ in $g_{1}$ such that

(a) two bounded elements of $H_{i}$ are adjacent if and only if the corresponding element of $G_{i}$ in $g_{1}$ are adjacent and

(b) corresponding elements of $H_{i+1}$ and $G_{i+1}$ are subsets of corresponding elements of $H_{i}$ and $G_{i}$.

(1.9) $T_{i}$ is a homeomorphism of the boundary of the sum of the elements of $G_{i}$ in $g_{1}$ into the boundary of the sum of the elements of $H_{i}$ such that $T_{i}$ preserves $T_{i-1}$ on the boundary of the sum of the elements of $G_{i-1}$ in $g_{1}$ and $T_{i}$ carries the boundary of an element of $G_{i}$ in $g_{1}$ into the boundary of the corresponding element of $H_{i}$.

Although the diameters of the bounded elements of $H_{i}$ may not approach 0 as $i$ increases without limit, $H_{1}, H_{2}, \cdots$ will satisfy a condition like (5.1) because $G_{1}, G_{2}, \cdots$ is a decreasing sequence of partitionings and $T_{i+n}$ preserves $T_{i}$ on the sum of the boundaries of the elements of $G_{i}$ in $\bar{g}_{1}$.

We find from Theorem 5 that there is an integer $n_{1}$ and a homeomorphism $F_{1}$ of $E^{3}$ into itself such that $F_{1}$ leaves each point of the exterior of $C$ in- 
variant and $F_{1}$ carries each bounded element of $H_{n_{1}}$ into a set of diameter less than $1 / 2$. We let $K_{1}$ be the partitioning of $E^{3}$ whose elements are the images of the elements of $H_{n_{1}}$ under $F_{1}$.

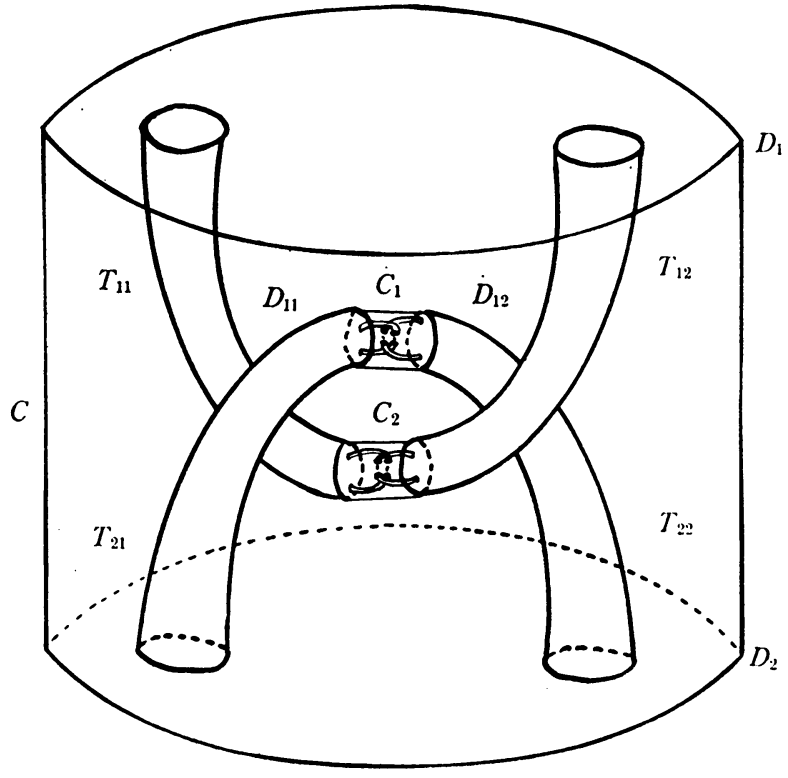

FIG. 4

An application of Theorem 5 to the elements of $K_{1}$ reveals that there is an integer $n_{2}$ and a homeomorphism $F_{2}$ of $E^{3}$ into itself such that (1) if $p$ is a boundary point of an element of $K_{1}, p$ is invariant under $F_{2}$, and (2) if $h$ is a bounded element of $H_{n_{2}}$, the diameter of the image of $h$ under $F_{2} F_{1}$ is less than 1/4. We denote the collection of images of elements of $H_{n_{2}}$ under $F_{2} F_{1}$ by $K_{2}$. If $T_{n_{1}}$ is the transformation mentioned in (1.9), we note that $F_{1} T_{n_{1}}$ is a homeomorphism of the boundary of the sum of the elements of $G_{n_{1}}$ in $g_{1}$ into the boundary of the sum of the elements of $K_{1}$ and that $F_{2} F_{1} T_{n_{2}}$ is a homeomorphism of the boundary of the sum of the elements of $G_{n_{2}}$ in $g_{1}$ into the boundary of the sum of the elements of $K_{2}$. Furthermore, $F_{2} F_{1} T_{n_{2}}$ preserves $F_{1} T_{n_{1}}$ on these boundaries.

Similarly, we find that there are integers $n_{3}, n_{4}, \cdots$ and partitionings $K_{3}, K_{4}, \cdots$ of $E^{3}$ satisfying conditions like conditions (1.5), (1.6), (1.7), and (1.8) satisfied by $H_{3}, H_{4}, \cdots$ and such that each bounded element of $K_{i}$ is of diameter less than $1 / 2^{i}$.

We find from Theorem 6 that these conditions imply that $\bar{g}_{1}$ and $C$ plus its interior are homeomorphic. A similar line of argument shows that $\bar{g}_{2}$ $+\bar{g}_{3}+\cdots+\bar{g}_{n}$ is also homeomorphic to $C$ plus its interior. Since each of these correspondences relates $F\left(g_{1}\right)$ to $C, S$ is topologicallyequivalent to $S^{3}$. 
Possible strengthening of Theorem 1. It may be noted that in neither Theorem 3 nor in Theorem 5 did we use the full strength of condition (1.2). Hence, at the expense of conciseness, a strengthening of the sufficiency part of Theorem 1 could be made here. For purposes of application in Theorem 1, condition (3.2) deals with $F\left(g^{\prime}\right) \cdot F\left(g^{\prime \prime}\right)$ where $g^{\prime}, g^{\prime \prime}$ are elements of $G_{i+1}$ in the same element of $G_{i}$; condition (4.2) deals with $F\left(g^{\prime}\right) \cdot F\left(g^{\prime \prime}\right)$ where $g^{\prime}, g^{\prime \prime}$ are elements of different $G_{i}$ 's and $g^{\prime}$ contains $g^{\prime \prime}$.

Consider the right cylinder $C$ with bases $D_{1}$ and $D_{2}$. The interiors of two mutually exclusive discs in $D_{i}(i=1,2)$ are replaced by the surfaces of tubes $T_{i 1}$ and $T_{i 2}$ and discs $D_{i 1}$ and $D_{i 2}$ as shown in Figure 4 where $D_{i 1}$ and $D_{i 2}$ are the bases of a right circular cylinder $C_{i}$ and $D_{1}+T_{11}+C_{1}+T_{12}$ is "hooked" to $D_{2}+T_{21}+C_{2}+T_{12}$ as shown. Discs in the bases of the cylinders $C_{1}$ and $C_{2}$ are replaced by surfaces of tubes and discs as before. If the process is continued and the limit taken, a wild simple surface $M$ is obtained. If $g$ is an element of $G_{1}$ and condition (1.2) is replaced by one like (3.2), $M$ might be the image of $F(g)$ in a homeomorphism of $S$ into $S^{3}$ if $S$ is topologically equivalent to $S^{3}$ and the elements of $G_{2}, G_{3}, \ldots$ in $g$ are fitted together as suggested after Theorem 4 in Figure 3. In fact, if $M_{1}$ and $M_{2}$ are two Alexander "horned spheres" $\left.{ }^{4}\right)$ in $S^{3}, T$ is a homeomorphism of $M_{1}$ into $M_{2}$, and $U_{i}$ $(i=1,2)$ is one of the complementary domains of $M_{i}$, then $S$ may be topologically equivalent to $U_{1}+M_{1}+U_{2}$ where it is understood that a point $p$ of $M_{1}$ is a limit point of a subset $V$ of $U_{2}$ if and only if $T(p)$ is a limit point of $V\left({ }^{5}\right)$.

Condition (1.3) prevents $S$ from being the sum of three 3-cells such that the common part of any two is the boundary of each. However, Theorem 1 would be somewhat stronger if the expression "1-dimensional" could be replaced by "0-dimensional or 1-dimensional."

Condition (1.4) rules out many 3-manifolds (as well as some spaces that are not 3-manifolds). It imposes a type of sequential unicoherence. However, unicoherence alone does not appear to be strong enough because projective 3 -space has a decreasing sequence of regular partitionings satisfying conditions (1.1), (1.2), and (1.3). One might wonder if simple connectedness might be substituted for condition (1.4).

Since $E^{3}$ is topologically equivalent to $S^{3}$ minus a point, the following is immediately obtained from Theorem 1.

Theorem 7. A metric space $S^{\prime}$ is topologically equivalent to $E^{3}$ provided it has a sequence of regular partitionings $G_{1}, G_{2}, \cdots$ satisfying conditions (1.1), (1.2), (1.3), and (1.4) as well as the following: $G_{i+1}$ is a refinement of $G_{i}$; exactly one element of $G_{i}$ fails to have a compact closure; for each point $p$ there is an

(4) J. W. Alexander, An example of a simply connected surface bounding a region which is not simply connected, Proc. Nat. Acad. Sci. U.S.A. vol. 10 (1924) pp. 8-10.

(5) R. L. Wilder, Topology of manifolds, Amer. Math. Soc. Colloquium Publications, vol. 32, New York, 1949, Problem 4.6, p. 383. 
integer $n(p)$ such that $p$ does not belong to the closure of this exceptional element of $G_{n(p)}$; the diameters of the elements of $G_{i}$ other than this exceptional element approach 0 as $i$ increases without limit.

Characterization of a simple closed curve. Since a 1-manifold without boundary is a simple closed curve, a simple closed curve is characterized by its local properties. Hence the following characterization of a simple closed curve is shorter than the corresponding characterizations of a simple surface and a 3 -sphere.

Theorem 8. A necessary and suffcient condition that $S$ be a simple closed curve is that one of its decreasing sequences of regular partitionings $G_{1}, G_{2}, \ldots$ have the following properties:

(8.1) The boundary of each element of $G_{i}$ is a pair of distinct points.

(8.2) No three elements of $G_{i}$ have a boundary point in common.

In fact, each decreasing sequence of partitionings $H_{1}, H_{2}, \cdots$ of a simple closed curve has properties (8.1) and (8.2) if $H_{1}$ has more than one element. Condition (8.2) could be replaced by the condition that the partitionings are brick partitionings.

Characterization of a simple surface. When a simple closed curve is partitioned into more than two elements, the boundary of each element of the partitioning is a pair of points. However, when a simple surface is partitioned there is a bigger variety of possible boundaries. They may either be simple closed curves or the sum of a finite number of simple closed curves.

TheOREM 9. A necessary and sufficient condition that $S$ be a simple surface is that one of its decreasing sequences of regular partitionings $G_{1}, G_{2}, \ldots$ have the following properties:

(9.1) The boundary of each element of $G_{i}$ is a simple closed curve.

(9.2) The intersection of the boundaries of 3 elements of $G_{i}$ contains no arc.

(9.3) If $g$ is an element of $G_{i-1}(g=S$ if $i=1)$ the elements of $G_{i}$ in $g$ may be ordered $g_{1}, g_{2}, \cdots, g_{n}$ so that $F\left(g_{j}\right)[j=1$ (if $\left.i>1), 2, \cdots, n\right]$ intersects $F(g)+G\left(g_{1}\right)+\cdots+F\left(g_{j-1}\right)$ in a nondegenerate connected set.

Indication of proof. Conditions (9.1), (9.2), and (9.3) correspond to conditions (1.1), (1.3), and (1.4) of Theorem 1. The word "nondegenerate" in condition (9.3) replaces the equivalent of condition (1.2) of Theorem 1. This condition cannot be deleted entirely or else $S$ could be the sum of two simple surfaces with only one point in common.

To prove Theorem 9, prove a theorem resembling Theorem 3 showing that there are a sequence of partitionings $H_{1}, H_{2}, \cdots$ of $S^{2}$ such that $H_{i+1}$ refines $H_{i}$ and a sequence of homeomorphisms $T_{1}, T_{2}, \cdots$ such that $T_{i}$ carries the boundary of the sum of the elements of $G_{i}$ into the boundary of the sum of the elements of $H_{i}$ and $T_{i+1}$ preserves $T_{i}$. Next prove a result like Theorem 5 
showing that there is no loss of generality in supposing that the maximum of the diameters of the elements of $H_{i}$ approaches 0 as $i$ increases without limit. Finally, use Theorem 6 to establish a homeomorphism between $S$ and $S^{2}$.

The following result throws some light on the role of condition (9.3). It follows from the fact that for continua $H, K$ in $S^{2}, S^{2}-(H+K)$ is not connected if $H \cdot K$ is not connected.

Theorem 10. Suppose $G_{1}$ and $G_{2}$ are two partitionings of $S^{2}, G_{2}$ refines $G_{1}$, the boundaries of the elements of $G_{1}$ and $G_{2}$ are connected, and $g$ is either $S^{2}$ or an element of $G_{1}$ whose boundary is nondegenerate. Then the elements of $G_{2}$ in $g$ may be ordered $g_{1}, g_{2}, \cdots, g_{n}$ such that $F\left(g_{j}\right)$ intersects $F(g)+F\left(g_{1}\right)+\cdots+$ $+F\left(g_{j-1}\right)$ in a nondegenerate connected set.

It would be interesting to know what the corresponding result is for $S^{3}$. In particular, if $G_{1}, G_{2}, \cdots$ are partitionings of $S^{3}$ satisfying conditions (1.1), (1.2), and (1.3), would they automatically satisfy condition (1.4)?

UNIVERSITY OF Virginia,

Charlottesville, Va.

UNIVERSITY OF WISCONSIN, MADISON, WIS. 